\title{
Culture de Pleurotus tuber-regium (Fr.) Singer sur substrat ligno-cellulosique en République Démocratique du Congo
}

Mwinyi Waziri, Lebisabo Bungamuzi, Kanyama Joseph, Rammeloo Jan, NshimbaSeya Wa Malale \& Degreef Jérôme

\section{Mwinyi Waziri :}

Université de Kisangani, Faculté des Sciences, République Démocratique du Congo

Auteur correspondant : kiumbewaziri@gmail.com

\section{Lebisabo Bungamuzi :}

Université de Kisangani, Faculté des Sciences, République Démocratique du Congo

\section{Kanyama Joseph :}

Université de Kisangani, Faculté de gestion des Ressources Naturelles Renouvelables, République Démocratique

Rammeloo Jan : Jardin botanique de Meise, Belgique

\section{NshimbaSeya Wa Malale :}

Université de Kisangani, Faculté des Sciences, République Démocratique du Congo

Degreef Jérôme : Jardin botanique de Meise, Belgique

Service général de l'enseignement supérieur et de la recherche scientifique, Fédération WallonieBruxelles, Belgique

DOI: $10.25518 / 2295-8010.1695$

\section{Résumé :}

Cette étude a concerné la culture sur pailles de riz et sciure de bois de Gilbertiodendron dewevrei de Pleurotus tuber-regium, espèce fongique tropicale appréciée pour sa saveur et ses propriétés médicinales. Les blancs ont été obtenus localement par isolement de spores de spécimens récoltés dans leur environnement naturel aux environs de Kisangani (RD Congo). La formation de sporophores est conditionnée à celle de sclérotes 14 jours au préalable. Trois poussées ont été enregistrées avec un rendement total de 42,25\% qui peut être considéré comme très satisfaisant et de loin supérieur au rendement économique de 20 \% généralement reconnu pour qu'un substrat soit jugé approprié à la production de champignons.

\footnotetext{
Abstract :

This study concerned the cultivation on rice straw and Gilbertiodendron dewevrei sawdust of Pleurotus tuber-regium, a tropical fungal species appreciated for its flavour and its medicinal properties. The spawns were obtained locally by isolating spores from specimens collected in their natural environment around Kisangani (DR Congo). The formation of sporophores is conditional to the formation of sclerotia 14 days beforehand. Three outbreaks were recorded with a total yield of $42.25 \%$, which can be considered very satisfactory and far above the generally accepted economic yield of $20 \%$ for a substrate to be considered suitable for
} 
Culture de Pleurotus tuber-regium (Fr.) Singer sur substrat ligno-cellulosiqu...

mushroom production.

\section{Introduction}

Les champignons sauvages comestibles jouent un rôle important dans la survie des populations rurales en Afrique tropicale. Ils sont particulièrement recherchés pour leur valeur nutritive élevée ainsi que leur valeur marchande, et près de 300 espèces y sont consommées par les populations locales (Rammeloo \& Walleyn, 1993 ; FAO, 2006 ; De Kesel \& Malaisse, 2010 ; Eyi et al., 2011 ; Degreef \& De Kesel, 2017). De nombreuses espèces de champignons sauvages sont récoltées au rythme d'apparition de leurs sporophores dans leur milieu naturel, généralement durant quelques semaines en période pluvieuse.

Des études taxonomiques permettent d'inventorier progressivement la diversité de ces champignons comestibles africains mais très peu de souches sauvages ont, jusqu'ici, fait l'objet d'essais de mise en culture, particulièrement en Afrique centrale (Degreef et al., 2016; Diansambu et al., 2015 ; Dibaluka et al., 2010 ; Yongabi, 2014). Pleurotus tuber-regium (Fr.) Singer est une espèce comestible saprotrophe, dont le cycle de vie est associé au développement d'un sclérote souterrain globuleux qui peut atteindre jusqu'à $30 \mathrm{~cm}$ de diamètre. Ce caractère très rare chez les macromycètes en permet une identification immédiate et ne prête à aucune confusion. Bien que son rattachement au genre Pleurotus ait été confirmé par des études moléculaires (Hitoshi \&Takao, 1995; Njouonkou, 2011), de nombreux auteurs persistent à maintenir l'espèce sous Lentinus tuberregium Sing. (Zmitrovich \& Kovalenko, 2016) en raison de ses caractères macroscopiques plus proches de ceux des lentins que des pleurotes, et notamment par la présence de tissus dimitiques. L'origine australasienne de Pleurotus tuber-regium a été révélée par l'étude phytogéographique d'Isikhuemhen et al. (2000).

Elle est ainsi rapportée d'Australie et de Papouasie-Nouvelle Guinée, mais également de Madagascar, d'Inde, du Sri Lanka, des Iles Salomon et de nombreux pays d'Afrique où elle est très appréciée comme aliment (Pegler, 1983).

En Afrique tropicale, l'espèce est signalée dans tous les types de forêts et dans les plantations, notamment au Bénin, au Burundi, au Cameroun, en République du Congo, en République démocratique du Congo, en Côte d'Ivoire, au Gabon, au Ghana, en Guinée, au Kenya, au Liberia, au Nigéria, en Ouganda, en Sierra Leone, en Tanzanie, au Tchad, en Zambie et au Zimbabwe (Degreef \& De Kesel, 2017). Outre les qualités nutritionnelles du sporophore (Apetorgbor et al., 2013 ; Okhuoya \& Isikhuemhen,1999), le sclérote est également utilisé à des fins thérapeutiques et est alors généralement réduit en poudre après séchage (De Kesel et al., 2017 ; Eyi et al., 2011; Isikhuemhen \& Nerud,1999).

Des études biochimiques ont ainsi mis en évidence la présence de composés actifs tels que des flavonoïdes, des phénols, différents alcaloïdes, des glycosides, des protéines et des enzymes dans le sclérote (Apetorgbor et al., 2013 ; Chenghua et al., 2000; Fasidi \& Ekuere, 1993 ; Isikhuemhen \& Nerud, 1999 ; Nwokolo, 1987 ; Wang \& Ng, 2001 ; Zhang et al., 2001). Elles ont également révélé son intéressant pouvoir anti-oxydant (Sukumar etal., 2018).

Au Nigéria, les médecins utilisent ces sclérotes pour la préparation d'un remède qu'ils administrent aux femmes enceintes afin d'aider le développement du fœtus (Oso, 1977). Au Ghana, ce même 
sclérote est utilisé pour soulager les maladies liées à la malnutrition et à l'anémie chez les enfants (Isikhuemhen \& Okhuoya, 1995). Le sclérote aurait également des pouvoirs coagulants et désinfectants (Chengua et al., 2000 ; Yongabi et al., 2011).

Un compte rendu ethnographique sur les bénéfices de la consommation de ce champignon a été publié par Baeke (2005). Le mycélium de Pleurotus tuber-regium aurait même la capacité d'améliorer les sols pollués aux hydrocarbures (Adenipekun, 2008 ; Isikhuemhen et al., 2003).

Une technique empirique de culture de Pleurotus tuber-regium est pratiquée dans la plupart des pays où l'espèce est signalée. Elle consiste à repérer la présence de l'espèce aux sporophores qui émergent du sol, à creuser quelques dizaines de centimètres et à prélever le volumineux sclérote souterrain qui est alors ramené au village et généralement enterré dans un endroit frais, humide et ombragé (Zadrazil \& Kurtzman, 1982).

Cette pratique culturale a été adoptée avec succès par des chercheurs nigérians afin de maximiser la production de sporophores de Pleurotus tuber-regium en conditions contrôlées (Fasidi \& Olorunmaye, 1994 ; Isikhuemhen \& Okhuoya, 1995,1996; Oghenekaro et al., 2008 ; Okhuoya \& Etugo, 1993 ; Okhuoya \& Okogbo, 1990, 1991 ; Okhuoya et al., 1998). Elle présente néanmoins l'inconvénient majeur de faire dépendre la mise en œuvre de la culture de la disponibilité de sclérotes prélevés en milieu naturel et dont la capacité à fournir des sporophores diminue en cours de production en raison de l'épuisement de leurs ressources.

Par ailleurs, en déterrant un sclérote et en l'utilisant comme semence, aucune garantie n'est donnée au producteur que la productivité de cette souche sauvage sera intéressante. Pour faire face à ces contraintes, la présente étude a opté pour une technique d'isolement de souche de Pleurotus tuber-regium à partir de spores, suivie de la production de blanc de semis. Cette méthode présente l'avantage de permettre de sélectionner et de conserver sur milieu gélosé les souches les plus performantes, tant au niveau de la croissance mycélienne que de leur capacité à produire des quantités importantes de sporophores.

Une technique apparentée, consistant à prélever un fragment de sclérote pour produire du blanc de semis, a précédemment été adoptée lors d'une étude dont l'objectif principal était d'estimer la valeur nutritive des sporophores de Pleurotus tuber-regium (Apetorgbor et al., 2013).

\section{Matériel et Méthodes}

\section{Isolement de la souche et mise en culture}

Un sporophore frais (spécimen P0252019Kis conservé en herbier à l'Université de Kisangani) a servi de matériel de référence pour l'obtention de spores. Un fragment du chapeau du spécimen frais est prélevé et fixé à l'ouverture d'un tube à essai à l'aide de ruban Parafilm, face hyméniale dirigée vers le milieu de culture PDA (filtrat de cuisson de $200 \mathrm{~g}$ de pommes de terre $+20 \mathrm{~g}$ d'agaragar $+20 \mathrm{~g}$ de dextrose porté à $1 \mathrm{l}$ et dilué dans $1 \mathrm{l}$ d'eau déminéralisée) (Dibaluka, 2005) (Figure 1). Le tube est mis à incuber à l'obscurité et à température ambiante durant 12 à 14 jours jusqu'à envahissement complet du milieu gélosé par le mycélium. 
Culture de Pleurotus tuber-regium (Fr.) Singer sur substrat ligno-cellulosiqu...

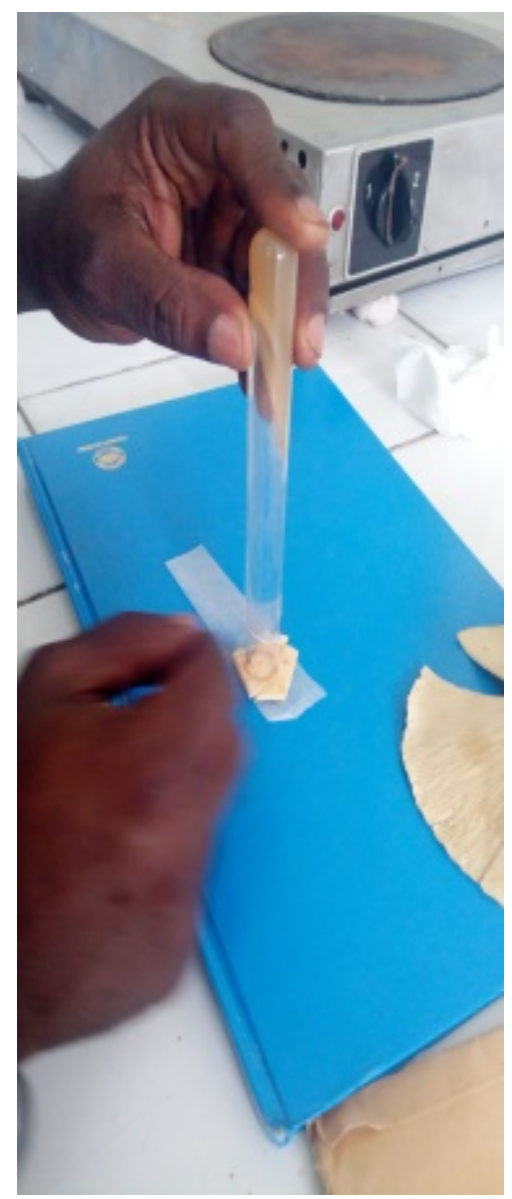

Figure 1 : Isolement de souche à partir d'une sporée

Le substrat de semis est préparé à partir de grains de sorgho traités comme suit : nettoyage à l'eau propre, cuisson (15 min), égouttage, ajustement du $\mathrm{pH}$ voisin de la neutralité avec de la chaux éteinte ( $2 \%$ ), répartition du milieu ( $300 \mathrm{~g})$ dans des bocaux fermés par un tampon d'ouate placé sous un couvercle vissé, stérilisation à l'autoclave $\left(120^{\circ} \mathrm{C}, 1 \mathrm{~h}, 1 \mathrm{~atm}\right)$.

L'inoculation du substrat de semis avec le mycélium de la culture pure prélevé sur milieu gélosé est réalisée dans des conditions de stricte asepsie sous flux laminaire, à côté d'une flamme à gaz.

L'incubation des grains de sorgho est menée dans une armoire aérée à l'obscurité $\left(27-29^{\circ} \mathrm{C}, 15\right.$ jours).

Le substrat de fructification constitué de pailles de riz et de sciure de bois de Gilbertiodendron dewevrei est préparé et mis à tremper dans l'eau pendant $24 \mathrm{~h}$. Il est ensuite égoutté et mis à fermenter sous bâche pendant 10 jours, puis composté pendant 30 jours. La teneur en eau du substrat de fructification avoisine alors $65 \%$. 


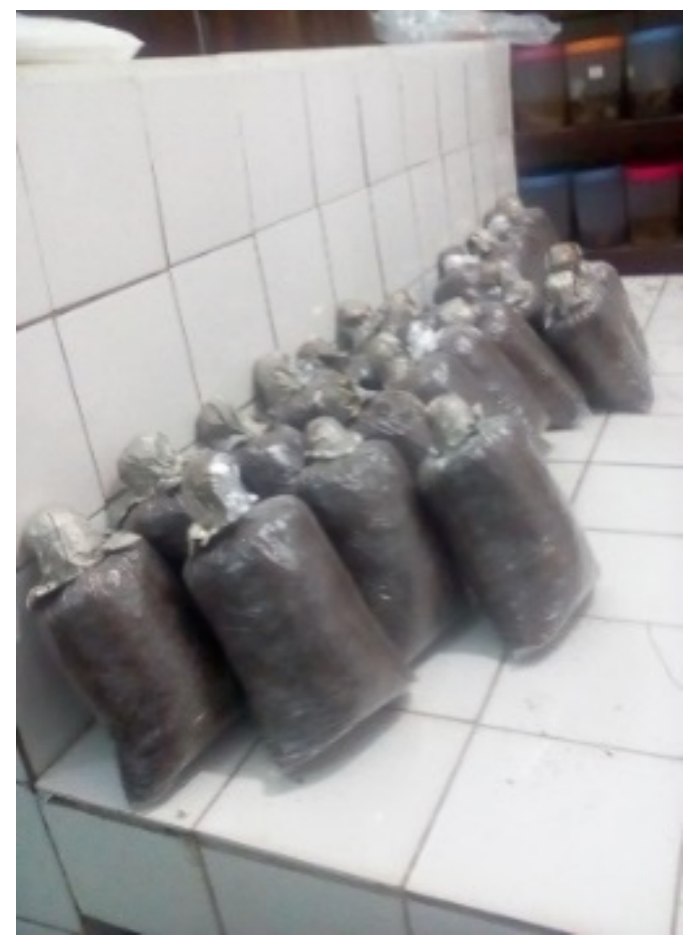

Figure 2 : Ballots fructifères avec anneau de $3 \mathrm{~cm}$ à l'encolure bouché d'ouate et couvert de papier aluminium.

La confection des ballots de fructification est réalisée par remplissage de sacs en plastique thermorésistants et doublés $(19 \times 28 \mathrm{~cm})$ contenant $600 \mathrm{~g}$ de substrat (figure 2 ) dont la composition est donnée au tableau 1 . Les sacs sont ensuite pasteurisés à l'autoclave à $120^{\circ} \mathrm{C}$ pendant $1 \mathrm{~h} 20$ min sous une pression de $1 \mathrm{~atm}$.

Tableau 1 : Composition des substrats de fructification des ballots fructifères

\begin{tabular}{lcc}
\hline Substrat & Poids (g) & Proportion (\%) \\
\hline paille de riz & 6000 & 68 \\
\hline sciure de bois & 1200 & 20 \\
\hline son de riz & 600 & 10 \\
\hline Gypse & 120 & 2 \\
\hline
\end{tabular}

Le lardage du substrat est réalisé $24 \mathrm{~h}$ après pasteurisation, en conditions aseptiques sous flux laminaire, à raison d'une cuillère à café de blanc de semis par ballot de fructification. Les ballots sont ensuite incubés dans des armoires à température ambiante $\left(28^{\circ} \mathrm{C}\right)$ et dans l'obscurité. L'uniformisation des conditions d'incubation est assurée par un déplacement aléatoire des ballots à l'intérieur de l'armoire, deux fois par semaine. L'incubation se poursuit avec l'envahissement 
Culture de Pleurotus tuber-regium (Fr.) Singer sur substrat ligno-cellulosiqu...

total du substrat de production par le mycélium suivi de l'apparition des sclérotes. La technique du gobetage est utilisée pour la phase de production. Les ballots de fructification contenant les sclérotes sont incisés et placés dans des caisses en bois puis enfouis sous de la terre meuble.

\section{Analyse des données}

La vitesse de croissance du mycélium est estimée par mesurage, à l'aide d'une règle graduée, de l'envahissement du milieu de culture. Elle est calculée sur le milieu de culture gélosé, sur le substrat de semis ainsi que sur le substrat des ballots de fructification.

La production de 17 ballots de fructification est évaluée par pesée du poids frais des sporophores produits par ballot lors de chacune des trois levées successives. L'analyse pour la comparaison des moyennes est réalisée sur les données des trois levées. Le rendement de production est estimé en pourcentage du poids total de sporophores frais obtenu par rapport au poids total de substrat des ballots de fructification.

Un test ANOVA est utilisé pour comparer les moyennes de production et un test de Tukey pour réaliser une comparaison multiple des moyennes.

\section{Résultats}

\section{Croissance du mycélium}

La vitesse moyenne de croissance du mycélium est de 0,24 $\pm 0,11 \mathrm{~cm} / \mathrm{jour}$ sur milieu PDA, de 0,75 $\pm 0,29 \mathrm{~cm} /$ jour sur le substrat de semis constitué de grains de sorgho et de 0,90 $\pm 0,33 \mathrm{~cm} / \mathrm{jour}$ sur le substrat des ballots de fructification.

\section{Productivité en sporophores}

L'envahissement complet des ballots de fructification par le mycélium est observé après 27 jours d'incubation. Quatorze jours s'écoulent ensuite avant la formation des sclérotes. La première levée survient 10 jours après la mise en terre des ballots fructifères contenant les sclérotes (Figure 3). La récolte est réalisée 3 à 4 jours après l'apparition des sporophores car ceux-ci deviennent rapidement coriaces et leur appétence en est diminuée. Deux autres levées se succèdent à un intervalle d'environ 30 jours. 


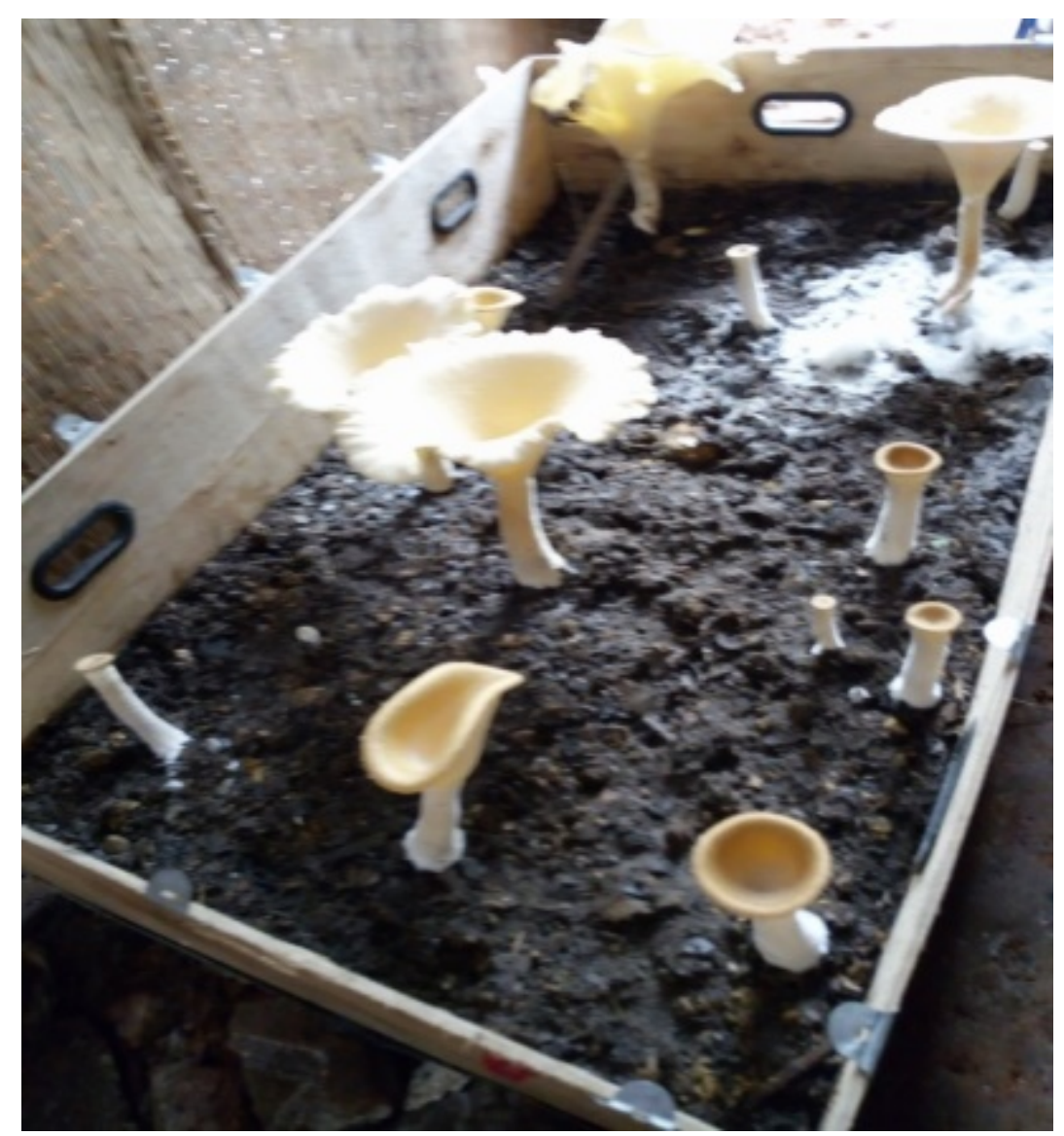

Figure 3 : Production de sporophores de Pleurotus tuber-regium par technique de gobetage

La productivité en sporophores est détaillée au tableau 2.

Tableau 2 : Productivité en sporophores (g) de 17 ballots de 600 g chacun

\begin{tabular}{llcc}
\hline & Levée 1 & Levée 2 & Levée 3 \\
\hline Poids total $(\mathrm{g})$ & 2354,4 & 1317 & 638,6 \\
\hline Poids moyen (g) par ballot & $138,49 \pm 45,90$ & $77,47 \pm 28,33$ & $37,56 \pm 24,88$ \\
\hline Rendement (\%) & $23,08 \pm 0,55$ & $12,91 \pm 0,31$ & $6,26 \pm 0,15$ \\
\hline
\end{tabular}

À l'issue des trois levées, les 17 ballots de fructification de $600 \mathrm{~g}$ (soit $10.200 \mathrm{~g}$ de substrat) ont produit un total de $4310 \mathrm{~g}$ de sporophores frais, soit un rendement total de 42,25\%.

Le poids le plus élevé est enregistré lors de la première levée et diminue ensuite jusqu'à l'épuisement du substrat. Le rendement par levée est obtenu en divisant le poids total de sporophores produits par le poids total du substrat. La comparaison des poids moyens obtenus par levée est illustrée dans la figure 4. 
Culture de Pleurotus tuber-regium (Fr.) Singer sur substrat ligno-cellulosiqu...

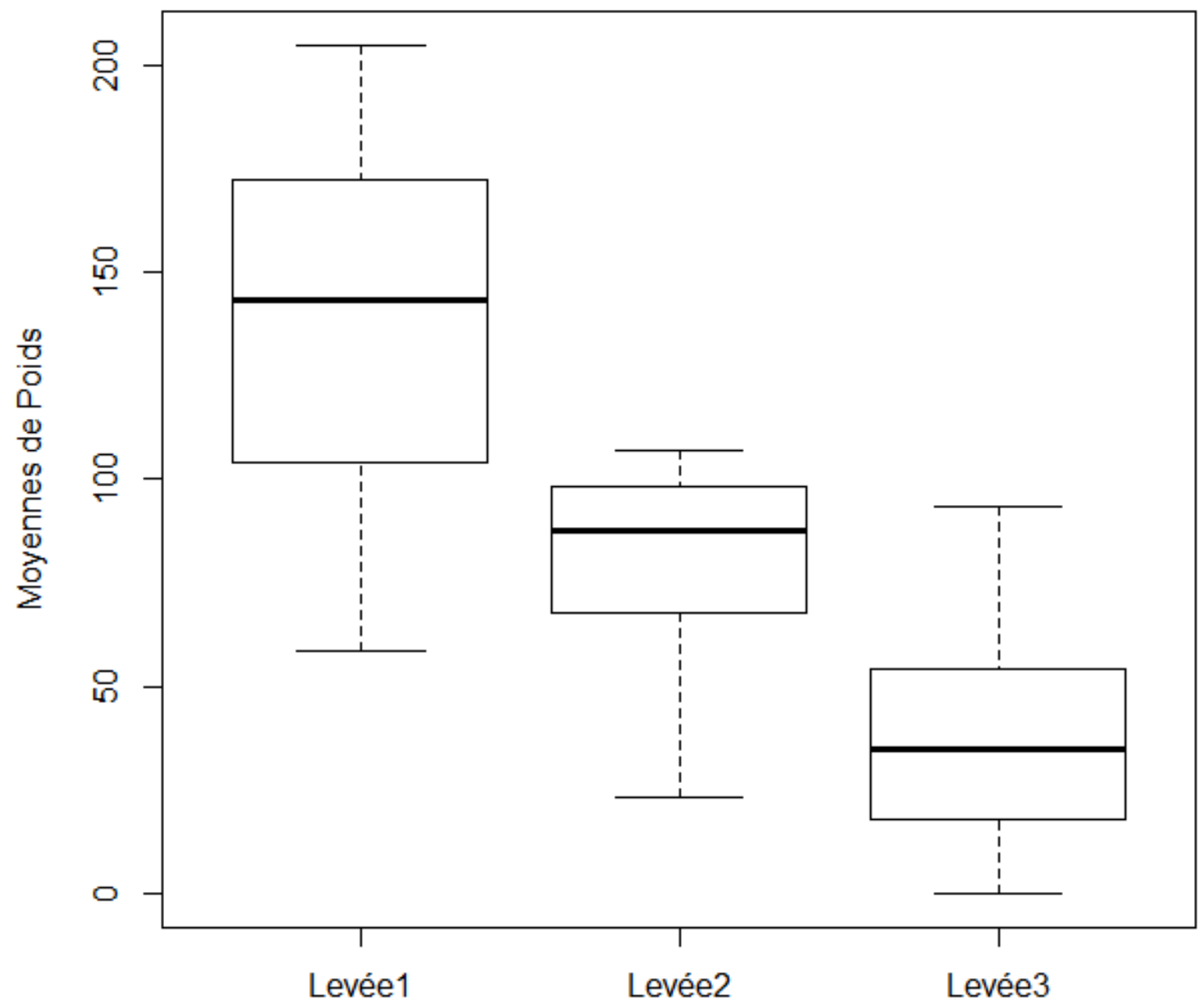

Figure 4 : Evolution du poids moyen en sporophores (g) par ballot au cours de la production.

Les tests de comparaison multiple des moyennes de production et des rendements de chaque levée révèlent qu'elles diffèrent significativement les unes des autres $(p<0,05)$, notamment en raison de l'épuisement du milieu, au fur et à mesure de la production.

\section{Conclusion}

La méthode de fructification de Pleurotus tuber-regium à partir de blanc de semis (obtenu non à partir de sclérotes mais par isolement de spores de spécimens récoltés dans leur environnement naturel) a donné des résultats prometteurs. Cette méthode ainsi que les résultats obtenus ouvrent des perspectives quant à la possibilité de sélectionner, en Afrique tropicale, des souches performantes de Pleurotus tuber-regium pour la mise en culture. Cet essai préliminaire, limité à une souche sauvage isolée dans les environs de Kisangani en République démocratique du Congo, a en effet démontré une vitesse de croissance du mycélium importante et des rendements de 
production en sporophores encourageants. Le rendement total de 42,25\% obtenu à l'issue de notre expérimentation peut en effet être considéré comme très satisfaisant et de loin supérieur au rendement économique de $20 \%$ généralement reconnu pour qu'un substrat soit jugé approprié à la production de champignons (Dibaluka et al., 2010 ; Oei, 2016). La réussite de la culture de Pleurotus tuber-regium apparaît très dépendante du choix du substrat comme en témoignent les échecs enregistrés sur feuilles de jacinthe d'eau (Eichhornia crassipes) et pailles de millet (Eleusine coracana) (Apetorgbor et al., 2013). Le mélange de riz paddy et de sciure de bois utilisé dans le présent essai constitue une alternative aux feuilles de bananier plantain ou à la sciure de bois compostée (Triplochiton scleroxylon) qui assurent également une production de sporophores de cette espèce (Apetorgbor et al., 2013). L'enjeu est désormais de réduire les délais entre les levées à travers une sélection de souches de diverses origines.

\section{Remerciements}

Le premier auteur remercie le Projet VLIR-UOS pour l'appui financier dans la réalisation de ce projet, les différentes formations en mycologie et algologie dont il a bénéficié ainsi que le soutien dans sa participation à différents symposiums sur la mycologie.

\section{Bibliographie}

1. Apetorgbor A.K., Dzomeku M. \&Apetorgbor M.M. (2013) Growth factors and cultivation of Pleurotus tuber-regium on selected plant wastes. International Food Research Journal 20(6): 3387-3393

2. Chenghua D., Xiangliang Y., Xiaoman G., Yan W., Jinggyan Z. \&Huibi X. (2000) A ß-Dglucanfrom the sclerotia of Pleurotus tuber-regium (Fr.) Sing. Carbohydrate Research 328: 629-633.

3. Degreef J. \& De Kesel A. (2017) The Edible Fungi of Tropical Africa annotated database [www.EFTA-online.org - accessed 15/05/2020]

4. Degreef J., Demuynck L., Mukandera A., Nyirandayambaje G., Nzigidahera B. \& De Kesel A. (2016). Wild edible mushrooms, a valuable resource for food security and rural development in Burundi and Rwanda. BiotechnologyAgronomy Society Environment 20(4): 441-452.

5. De Kesel A. \&Malaisse F. (2010). Edible Wild food: Fungi. In: Malaisse F. (ed.), How to live and survive in Zambezian Open Forest (Miombo Ecoregion). Gembloux (Belgium) : Presses Agronomiques de Gembloux : 41-56.

6. Diansambu M.I., Dibaluka M.S., Lumande K.J. \&Degreef J. (2015) Culture de trois espèces fongiques sauvages comestibles du Groupement de Kisantu (RD Congo) sur des substrats ligno-cellulosiques compostés. Afrique Science 11(3) : 241-261.

7. Dibaluka M,S., Lukoki F.L., De Kesel A. \&Degreef J. (2010) Essais de culture de quelques champignons lignicoles comestibles de la région de Kinshasa (R.D. Congo) sur divers substrats lignocellulosiques. BiotechnologyAgronomy SocietyEnvironment : 417-422.

8. Dibaluka M.S. (2005) Inventaire des macromycètes de la forêt de lac de Ma 
Culture de Pleurotus tuber-regium (Fr.) Singer sur substrat ligno-cellulosiqu...

Vallée(Kinshasa) et essai de mise en culture des quelques espèces comestibles, Mémoire (DEA) inédit, Département de Biologie, Faculté des Sciences, Université de Kinshasa, $75 p$.

9. EyiNdong H., Degreef J. \& De Kesel, A. (2011) Champignons comestibles des forêts denses d'Afrique centrale, Taxonomie et identification, Abc Taxa 10, 254p.

10. FAO (2006) Champignons comestibles sauvages. Vue d'ensemble sur leurs utilisations et leur importance pour les populations. Rome, Italie, 170p.

11. Fasidi I.A. \&Ekuere U.U. (1993) Studies on Pleurotus tuber-regium: Cultivation, proximate composition and mineral content of sclerotia. Food Chemistry 48: 255- 258.

12. Hitoshi N. \& Takao N. (1995) Phylogenetic analysis of Pleurotus based on data from partial sequences of 18rDNA and ITS-1 regions. Mushroom Science 14: 161-168.

13. Isikhuemhen O.S. \&Nerud F. (1999) Preliminary studies on the ligninolytic enzymes produced by the tropical fungus Pleurotus tuber-regium. Antonie van Leeuwenhoek 75 : 257-260.

14. Isikhuemhen O. S. \&Okhuoya J. A. (1995) A low cost technique for the cultivation of Pleurotustuberregium (Fr.) Singer in developing tropical countries. Mushroom Growers Newsletter 4, 2-4.

15. Isikhuemhen O.S. \&Okhuoya J.A. (1996) Cultivation of edible sclerotia of Pleurotus tuberregium (Fr.) Singer on agricultural wastes. InD.J. Royse (ed.): Proceedings of the 2nd International Conference on Mushroom Biology and Mushroom Products, 9-12 June 1996: 429-436. Pennsylvania State University, USA.

16. Isikhuemhen O.S., Moncalvo J.M., Nenid F.\&Vilgalys R. (2000) Mating compatibility andphylogeography in Pleurotustuberregium. Mycological Research. 104: 732-737.

17. Njouonkou A.L. (2011) Taxonomie, systématique et étude phylogénétique des genres Lentinus Fr. et Pleurotus (Fr.) Kramer au Cameroun basée sur la morphologie et les séquences des régions ITS de l'ADNr et identification des enzymes extracellulaires oxydases de quelques champignons lignivores. Thèse de Doctorat, Université de Yaoundé I, $198 \mathrm{p}$.

18. Nwokolo, E. (1987). Composition of nutrients in the sclerotium of the mushroom Pleurotus tuber-regium. Plant Foods and Human Nutrition 37: 133-139.

19. Oei P. (2016). Mushroom cultivation. $4^{\text {th }}$ edition. Eco Consult Foundation, The Netherlands, $520 \mathrm{p}$.

20. Oghenekaro A.O., Okhuoya J.A. \&Akpaja E.O. (2008) Growth of Pleurotustuberregium (Fr.) Singer on some heavy metal-supplemented substrates. African Journal Microbiology Research2:268-271.

21. Okhuoya J.A. \&Isikhuemhen O.S. (1999) Mushroom cultivation: The Nigerian Experience. In. A. Broderick, T. Nair. (Eds.) Proceedings of the 3rd International conference on 
Mushroom Biology and Mushroom Products \& AMGA's26th National Mushroom Industry Conference.October 12-16, 1999. Sydney: 380-389.

22. Okhuoya J.A. \&Etugo J.E. (1993) Studies on the cultivation of Pleurotus tuber-regium (Fr.) Sing., an edible mushroom. Bioresource Technology 44:1-3.

23. Okhuoya J.A. \&Okogbo F.O. (1990) Induction of edible sclerotia of Pleurotus tuber-regium in the laboratory. Annals of Applied Biology 117: 295-298.

24. Okhuoya J.A. \&Okogbo F.O. (1991) Cultivation of Pleurotus tuber-regium (Fr.) Sing. on various farm wastes. Proceedings of Oklahoma Academy of Science 71: 1-3.

25. Okhuoya J.A., Isikhuemhen O.S. \&Evue G.A. (1998) Pleurotustuberregium(Fr.) Sing.: Sclerotia and sporophore yieldduring cultivation on sawdust of different woody plants. The International Journal of Mushroom Science2 (2): 41-46.

26. Oso B.A. (1977) Pleurotus tuber-regiumfrom Nigeria.Mycologia 69:272-279.

27. Pegler D.N. (1983) The genus Lentinus: a world monograph. HMSO, London.

28. Rammeloo J. \&Walleyn R., (1993). The edible fungi of Africa south of the Sahara: a literature survey. Scripta Botanica Belgica 5: 1-62

29. Sukumar D., Mnoj K., Rakesh R. \&Manoranjan P.S. (2018) Pleurotus tuber-regium (Rumph. ex Fr.) Singer a potent source of antioxidant. Balneo Research Journal 9(3): 228-31. DOI: http://dx.doi.org/10.12680/balneo.2018.186

30. Wang H.X. \& Ng T.B. (2001) Isolation of pleuturegin, a novel ribonuclease-inactivating protein from the sclerotia of the edible mushroom Pleurotustuberregium. Biochemistry and Biophysics Research Communications 288:718-721.

31. Yongabi K.A. (2014). Current development in mushroom biotechnology in sub-Saharan Africa. Bulletin of the World Society for Mushroom Biology and Mushroom Products 11: 4-13.

32. Zhang M., Cheung P.C.K. \& Zhang L. (2001) Evaluation of mushroom dietary fiber (nonstarch polysaccharides) from sclerotia of Pleurotus tuber-regium (Fr.) Singer as a potential antitumour agent. Journal of Agriculture and Food Chemistry 49:5059-5062.

33. Zmitrovich I.V. \& Kovalenko A.E. (2016) Lentinoid and polyporoid fungi, two generic conglomerates containing important medicinal mushrooms in molecular perspective. International Journal of Medicinal Mushrooms 18(1):23-38.

PDF généré automatiquement le 2023-04-26 15:13:40

Url de l'article : https://popups.uliege.be/2295-8010/index.php?id=1695 